\title{
Alcoholism Fuels Gender-Based Violence in the Family
}

\author{
Pamela R. N. Kaithuru \\ Catholic University of Eastern Africa, Nairobi, Kenya
}

\begin{abstract}
Gender is the fact of being either male or female a fact used culturally to determine the roles or pattern of behavior and attitudes which either of the genders is expected to exhibit. Gender violence on the other hand refers to behavior "geared" to hurt the other depending on their "sex". There is a social construction of masculinity that condones male dominance over women with average of $50 \%$ of women in the world reporting being physically abused by intimate partners. Gender-based violence (GBV) is an issue of concern because it takes many forms and it is the most likely to happen. It encompasses physical, sexual, and psychological violence occurring in the family as well as in the general community. From a psychological perspective, GBV is an observed behavior. Alcohol abuse is a factor that fuels GBV in families. The research sought to investigate GBV that is related to alcoholism in families in Dagoret Constituency, Nairobi County in Kenya. The survey used descriptive research design. The data was collected by the use of semi-structured questionnaires and review of documents. The target population for this study was 100 gender based survivors from Dagoret who had experienced alcoholism in the last three years. The sample size of 30 participants was selected through purposive sampling. Cognizant with the principles of GBV research this study upheld pertinent ethical principles especially confidentiality and respect. Results from the study were analyzed using quantitative and qualitative techniques and interpretation presented in form of charts and tables. The study concluded that there is relationship between alcoholism and GBV in families in Dagoret Constituency and recommended awareness, counseling and legal redress as necessary.
\end{abstract}

Keywords: GBV (gender-based violence), family, alcoholism

\section{Introduction}

Gender is the fact of being either male or female a fact used culturally to determine the roles or pattern of behavior and attitudes which either of the genders is expected to exhibit. It refers to the different characteristics of men and women that are socially determined. Gender is not sex. The term "sex" refers to the different biological characteristics between males and females. Conversely, gender refers to the different social roles men and women have in a particular society. It defines culturally acceptable attitudes, behavior, responsibilities opportunities and constraints of men and women (WHO , 2012). "Gender is relational and refers not simply to women or men but to the relationship between them". Gender also refers to the economic, social and cultural attributes and opportunities associated with being male or female at a particular point in time. As Porter and Sweetman (2005) posits, gender refers to the socially constructed roles of and relations between men and women. Gender is also defined as the varying roles and attributes of men and women in diverse social, cultural and political contexts. "Gender refers to the array of socially constructed roles and relationships, personality traits, attitudes, behaviors, values, relative power and influence that society ascribes to the two sexes on a 
differential basis. Whereas biological sex is determined by genetic and anatomical characteristics, gender is an acquired identity that is learned, changes over time, and varies widely within and across cultures".

Violence on the other hand refers to all acts or threats that cause direct physical, mental or sexual harm or suffering. Research defines violence to also include indirect acts such as coercion and intimidation. It is pertinent to point out that whereas women, men, boys and girls can be victims of GBV, women and girls are disproportionately affected. In GBV literature, a victim of GBV is most often referred to as survivor (UNIFEM, 2008).

At the global level, research by WHO has strongly indicated that GBV is perpetrated by husbands or male partners (WHO, 2011; 2012). According to Kenya National Bureal of Stattistics (2009), GBV and forced sex are highly prevalent in the East African region. According to a research conducted in Kasarani, $43 \%$ of $15-49$ year old women reported having experienced some form of GBV in their lifetime, with $29 \%$ reporting an experience in the previous year; $16 \%$ of women reported having ever been sexually abused, and for $13 \%$, this had happened in the last year. The research found out that 47,551 women aged 15-49 are likely to have experienced physical violence since age 15 while 39,352 women aged 15-49 are likely to have experienced physical or sexual violence committed by a husband/partner (Otsola, 2012).

According to United Nations Declaration on Elimination of Violence among family members (1993) domestic violence against families is defined as any act of domestic violence that results in or is likely to result in physical, sexual or psychological harm or suffering to families including threats of such acts, coercion or arbitrary deprivation of liberty, whether occurring in public or in private domain (Sweetman, 1998).

GBV includes a variety of acts of violence committed against females because they are females and against males because they are males. It includes sexual violence, intimate partner or spouse abuse (domestic violence), emotional and psychological abuse, sex trafficking, forced prostitution, sexual exploitation, sexual harassment, harmful traditional practices and discriminatory practices based on gender. Research indicates that most GBV cases involve a female survivor and a male perpetrator. Research further indicates that the majority of acts of GBV against boys and men are also committed by male perpetrators (Beth, 2002). This is any rough and illegitimate use of physical force, aggression, or verbal abuse by one family member towards another. Study has revealed that each family is governed and homeostasis is maintained by rules either implicit or explicit with individual members playing roles within set family boundaries or patterns of relationships. In relations to alcoholism, where such violation occurs, alcohol use is explained as a maladaptive way of coping with or adapting to a dysfunction in the family (Oketch, 2008).

Domestic violence can be physical and/or psychological with statistics indicating that $95 \%$ of domestic violence is towards women (Insel \& Roth, 2002). More often than not the root course of this form of violence is desire to control and have power over the partner due to ones low self-esteem. The controlling partner may physically harm, arouse fear, prevent the other from expressing self and making them do things they do not want to.

Both men and women may experience GBV in our society. Those most vulnerable are women and children. A battered woman becomes vulnerable and sometimes even addicted to battering. They may flee initially and seek for help, but because of lowered self esteem, prefer going back to the perpetrator with a hope for change. During such a time, the perpetrator becomes remorseful and promises change. He may even become extra loving and the survivor decides to stay. After some time, the cycle is repeated. The cycle may only be broken through counseling if the survivor is determined to be helped (COVAW, 2001). On the other 
hand, our culture does not allow battering of men and when it occurs they do not cry for help. Only recently men are coming out in the open to share their plight.

\section{Alcohol Abuse and Alcoholism}

According to the Diagnostic Statistical Manual (DSM-IV), addiction is a chronic relapsing condition characterized by compulsive drug seeking and abuse that causes long-lasting chemical changes in the brain with the inability to stop use despite knowing the consequences it causes. Addiction is the repetitive, compulsive seeking and use of a substance, a disorder that has characteristics of loss of control in limiting intake, and emergence of negative emotional states which occurs despite known negative consequences that as it were results to psychological dependence (Miller \& Carroll, 2006) .

Global statistics indicate that well over 200 million people abuse drugs, with the most abused drug being alcohol, with impacts being felt differently in regions, communities and families. In Africa, abuse is more common among men than women, though trends are changing rapidly. Amongst women abuse is "less visible, more private" has it is viewed more as a male problem with women being more involved in the trade and being victims of the same. With urbanization and technological advancement though these trends are changing and alcohol abuse has become widespread both in rural and urban settings with learning institutions (tertiary), as evidenced in a report by NACADAA (2006), indicating increasing trends of areas where the young are initiated into substance abuse. Research findings by NACADA (2010) revealed that about 40\% of Kenyans aged 15-65 years have drank one type of alcohol or another, with at least $13 \%$ of people from all provinces in Kenya (except for North Eastern) consuming alcohol.

As NACADAA (2010) asserts, there is an estimated two million alcoholics in Kenya. Use of substances is higher amongst male than females with $25 \%$ of males in Nairobi province using legal alcohol as compared to $11 \%$ females. It is evident in the same report that $31.6 \%$ of urban men and women (15-64 years) are more likely to have consumed packaged/legal alcohol as compared to $21.6 \%$ in the rural areas. The report noted more access to traditional liquor, with urban average of $18 \%$ as compared to $13 \%$ in the rural areas.

It is estimated that one alcoholic intimately affects at least six persons. Study has revealed that $16 \%$ of full time employees have serious drinking problems with about $25 \%$ of hospitalized patients being related to alcohol, 40\% industrial accidents linked to alcohol abuse, 100,000 death annually occasioned by alcohol abuse and about 500 millions lost work days annually due to alcoholism.

\section{Statement of the Problem}

Research has identified links between alcohol abuse and the occurrence of violence in many countries. There is evidence to show that alcohol abuse increases the occurrence and severity of domestic violence. Directly alcoholism can create an unhappy and stressful family environment that increases the risk of conflicts and therefore violence.

Study by WHO shows that alcoholism affects cognition and physical functioning reducing self control and therefore leaving individuals less capable to negotiate non-violet resolutions of conflicts in relationships. It is also notable that alcoholism exacerbates financial difficulties, child care, infidelity and other family stressors. These create tension increasing the risk of violence in the family.

A number of individual, relationships and societal factors can exacerbate the association between alcohol abuse and violence. For alcoholics it increases the risk of violence and also victimization. There is evidence 
that poor mental health co-occurs with problematic alcohol use which is a risk factor for violet offending. Alcoholism is also related to antisocial personality disorders. In addition if leads to aggressive behavior hence increasing risk for violence in the family. Societal beliefs about alcohol abuse, gender roles and violent behavior towards female partners as masculinity further add to GBV. There are "socially" accepted violent behaviours which further fuels the GBV by individuals abusing alcohol. The impact associated with sexual and GBV revolves around all areas of social and health, legal and security programming. The areas include: increased rates of mortality and morbidity and exacerbated transmission of HIV among other health conditions.

Research has shown that the impact of violence on development progress goes beyond short-term injury and disability. The consequences of GBV include HIV and AIDS and Sexually Transmitted Infections, unwanted pregnancies, physical and psychological trauma. It has been established that GBV leads to the isolation and even ostracism of the victims, and ultimately, to longer-term mental, medical and economic consequences. Additionally, children of both sexes raised in a violent family will be shaped by the experience (USAID, 2009).

According to a report by the World Health Organization (2005), most divorced women normally site cases of GBV that lead to the divorce or separation. There is a correlation between sexual and GBV, health, human rights and national development in East, Central and Southern Africa. In Kenya, GBV has been reported and manifest in various forms with greater impacts in the individuals, families and communities. With the increase in alcohol abuse in the community needless to say it fuels the violence and therefore the need to investigate the link as is the aim of this research.

The study was guided by the following research questions: Is there gender violence in the family in Dagoret constituency? Does alcohol abuse contribute to GBV in the family in Dagoret County?

\section{Research Methodology}

Research design is the plan that is used to generate answers to the research problem (Mugenda \& Mugenda, 2003). The study adopted survey research design in order to provide a framework to examine current conditions, trends and status of the study phenomenon. Survey design being descriptive is more investigative and focuses on a particular variable factor. It is analytical and often singles out a variable factor or individual subject and goes into details and describing them. The researcher used descriptive research design to investigate domestic violence on family in relation to alcoholism.

This study targeted 100 persons from Dagoret constituency, Nairobi County. This is the number of estimated number of people that had experienced alcoholism in the past three years (Reports of Sub-County, 2012). The target population included men and women drawn from three villages in Kabete. A sample size of 30 Participants was targeted. This represents $30 \%$ of the target population. Purposive sampling technique was used to identify respondents for key players in gender violence related cases. Purposive sampling technique is normally used for choosing people whose views are relevant to the issue.

The survey involved the use of both primary and secondary data. The researcher used questionnaire, most widely used tool for collection obtaining information that cannot be easily observed but can be used for description, explanation-and hypothesis testing in research (Remenyi et al., 1998; Saunders et al., 2003). Semistructured interviews were also conducted for collecting in-depth information rely on facts that people are able to offer an account of in terms of behaviour, practices and actions to the researcher (Kumar, 2005). 
The study used quantitative and qualitative methods of data analysis. Secondary data was also used to support the findings. Tables, graphs and charts were used.

\section{Data Analysis}

Table 1

Sex of Respondents

\begin{tabular}{llc}
\hline Sex & Number & Percentage (\%) \\
\hline Male & 11 & 34.4 \\
Female & 21 & 65.6 \\
Total & 32 & 100 \\
\hline
\end{tabular}

Majority of the respondents accounting for $65.6 \%$ were female while male accounted for $34.4 \%$ respectively.

Table 2

Gender Violence

\begin{tabular}{lcl}
\hline Response & Frequency & Percentage (\%) \\
\hline "Yes" & 21 & 70 \\
"No" & 9 & 30 \\
Total & 30 & 100 \\
\hline
\end{tabular}

$70 \%$ of the respondents have experienced GBV in their homes while $30 \%$ have not experienced GBV in their homes.

Table 3

Reported Abuse

\begin{tabular}{lll}
\hline & Frequency & Percentage (\%) \\
\hline Verbal abuse & 17 & 56.6 \\
Physical abuse & 9 & 30.0 \\
Sexual abuse & 4 & 13.3 \\
Total & 30 & 99.9 \\
\hline
\end{tabular}

Majority of the participants, $56.6 \%$ had suffered verbal abuse while $30.0 \%$ has suffered physical abuse and a minority of $13.1 \%$ had suffered sexual abuse. The survey concluded that verbal abuse is highest form of violence manifest in this population.

Table 4

History of Alcohol Abuse

\begin{tabular}{llc}
\hline & Frequency & Percentage (\%) \\
\hline "Yes" & 18 & 60 \\
"No" & 12 & 40 \\
Total & 30 & 100 \\
\hline
\end{tabular}

Majority of the respondents accounting for $60 \%$ admitted that there was a history of alcohol abuse in their families while $40 \%$ said that there was no history of drug abuse in their families. The findings reveal most people in the population have a history of alcohol abuse over the years within Dagoret constituency. 
Table 5

Those Abusing Alcohol in the Family

\begin{tabular}{lcc}
\hline Gender & Frequency & Percentage (\%) \\
\hline Mother & 2 & 6.7 \\
Father & 11 & 36.6 \\
Brother & 5 & 16.6 \\
Sister & 1 & 3.3 \\
Self & 5 & 16.6 \\
Son & 6 & 20 \\
Total & 30 & 99.8 \\
\hline
\end{tabular}

$36.6 \%$ of the respondents indicate that their fathers' abuse alcohol, $20 \%$ of the sons while $16.6 \%$ said it was their brothers and themselves respectively. $6.7 \%$ of the mothers and $3.3 \%$ of the sisters of the participants abuse alcohol.

Table 6

Duration of Alcohol Abuse

\begin{tabular}{lll}
\hline Number of years & Frequency & Percentage (\%) \\
\hline 1 & 4 & 13.3 \\
10 & 12 & 40.0 \\
$11+$ & 14 & 46.7 \\
Total & 30 & 99.9 \\
\hline
\end{tabular}

Majority of respondents accounting for $46.7 \%$ agree that alcohol abuse has been going on for many years in their families. $40 \%$ admitted that alcohol abuse has been going on for one 10 years and $13.3 \%$ indicated that alcohol abuse has taken root in the last 1 year in the family respectively. The survey concluded that many respondents have lived with family members abusing alcohol for many years.

\section{Results}

Findings showed that respondents accounting for $65.6 \%$ were female while male accounted for $34.4 \%$ respectively. Of the respondents, $70 \%$ have experienced GBV in their homes while $30 \%$ have not experienced GBV in their homes. The findings indicated that most of the participants, $56.6 \%$ had suffered verbal abuse while $30.0 \%$ has suffered physical abuse and a minority of $13.1 \%$ had suffered sexual abuse. Majority of the respondents accounting for $60 \%$ admitted that there was a history of alcohol abuse in their families while $40 \%$ said that there was no history of drug abuse in their families. In this study, $36.6 \%$ of the respondents indicate that their fathers' abuse alcohol, $20 \%$ of the sons while $16.6 \%$ said it was their brothers and themselves respectively, $6.7 \%$ of the mothers and $3.3 \%$ of the sisters of the participants abuse alcohol. Findings also showed that $46.7 \%$ agree that alcohol abuse has been going on for more than 11 years in their families. $40 \%$ admitted that alcohol abuse has been going on for 10 years and $13.3 \%$ indicated that alcohol abuse has taken root in the last 1 year in the family respectively.

\section{Discussion}

As observed in this study alcohol abuse increases the occurrence of GBV in the family. It is evident that the abuse is directed towards the female gender by the males who also have a higher prevalence of alcohol abuse. Other quantitative and in-depth studies revealed not only the widespread experience of violence 
permeating women's lives, but also the range of perpetrators of this violence. Intimate partners and family members directed violent behavior towards women and more so verbally followed by physical abuse. This can be attributed to the fact that alcohol abuse and more so alcoholism impairs ones judgment and lowers ones inhibitions therefore perpetrating verbal and physical abuse. Reports of sexual abuse are low which could be linked to stigma related to this form of abuse, especially in the African context, making it difficult to verbalize and more so when it happens within the "couple" subsytem. As the research observed, currently the female gender is suffering the GBV, a fact that may change with time. This is so bearing in mind that gender is so pervasive that in our society we assume it is bred into our genes. Most people find it hard to believe that gender is constantly created and re-created out of human interaction, out of social life, and is the texture and order of that social life. Gender, like culture, is a human production that depends on everyone constantly "doing gender" (West \& Zimmerman, 1987). The fluid nature of the society then puts either gender at risk of being violated at one time or another, therefore, addressing gender based violence is key to ensuring that humanness is respected irrespective of gender per time.

\section{Limitation}

The limitation of this study was the time and also the sensitive of family's issues which are often times treated as normal and or secret such that individuals minimize them. Causality cannot be inferred from the findings only associations can be noted. In African communities or settings to speak openly about their lifetime experiences of matters that may be socially stigmatizing was also a limitation.

\section{Conclusion}

The survey observed that most of the respondents were female and that gender violence is high within Dagoret constituency. The survey concluded that verbal abuse is high in the constituency compared to other forms of abuse which could often be occurring under the guise of drunkenness. It was notable that fathers and sons were most of the alcoholics with long history of alcoholism being evidenced in the constituency. Further the study concluded that there is relationship between alcoholism and GBV perpetuated by males. The study recommends that necessary therapeutic measures, through probation office and local administration, like rehabilitations and awareness creation need to be put in place. In addition measures to offer counseling services for the affected families are recommended. The study further recommends the investigation of the effects of GBV in families in the region.

\section{References}

COVAW. (2001). Easter and Central Africa women in development Network, 2003.

Edwards, M. (1989). Crime and delinquency. Kenya Literature Bureau.

Esplen, E., \& Jolly, S. (2006). Bridge development gender: Gender and sex, a sample of definitions. Retrieved from http://www.bridge.ids.ac.uk

Insel, P., \& Roth, W. (2002). Core concepts in health (9th ed.). Boston: Mc-Graw Hill.

Kenya National Bureau of Statistics. (2009). Policy on drug and alcohol abuse. Nairobi: Government of Kenya Printers.

Miller, R. W., \& Carroll, M. K. (2006). Rethinking substance abuse; what the science shows, and what we should do about it. New York: The Guilford Press.

Mugenda, O. M., \& Mugenda, A. B. (2003). Research methods: Quantitative and qualitative approaches. Nairobi; Acts Press.

NACADA. (2010). Alcohol and drug abuse_Driving the HIV/AIDS epidemic. NACADAA.

NACADA. (2007). Rapid situation assessment of drug \& substance abuse in Kenya. NACADAA. 
National Campaign Against Drug Abuse. (2006). Drug and substance abuse in tertiary institutions in Kenya. Nairobi: NACADAA.

Otsola, J. K. (2012). Baseline survey report on gender based violence in Kasarani. Nairobi: Kenya Women and Children's Wellness Centre.

Porter, F., \& Sweetman, C. (2005). Mainstreaming gender in development: A critical review oxfam focus on gender. Oxfam.

Sweetman, E. (1998). Social problems of man in domestic violence. The Dorsey Press.

UNIFEM. (2008). An empirical analysis of cases of gender-based violence in Rutsiro, Kayonza, Ngororero Districts and the City Of Kigali. UNIFEM.

USAID. (2009). A guide to programming gender-based violence prevention and response activities. Gender-Based Violence Working Group USAID, USAID.

West, C., \& Zimmerman, D. H. (1987). Gender and Society, 1(2), 125-151.

World Health Organization. (2011). Global status report on alcohol and health. Geneva: WHO.

World Health Organization. (2012). Alcohol in the European Union: Consumption, harm and policy approaches. Copenhagen: WHO.

World Health Organization. (2003). Guidelines for medico-legal care for sexual violence victims. Geneva: WHO. 